\title{
On the Impact of Network QoS on Automated Distributed Auctions
}

\author{
Ricardo Lent \\ Intelligent Systems and Networks \\ Imperial College London, SW7 2AZ UK \\ r.lent@imperial.ac.uk
}

\begin{abstract}
Autonomic networks offer a promising technology for the development of future distributed services, which will benefit from self-adapting communications with consequent improvements in quality of service (QoS). As electronic commerce over shared computer networks continues to grow, it becomes meaningful to gain better understanding of the relationship between network QoS and the expected results of time-sensitive e-commerce applications. In particular, to quantify possible utility improvements with the introduction of autonomic networks. This work is a simulation study of a time critical e-commerce application in the form of a system of distributed auctions that relies on a computer network for marketing, matching sellers and buyers, and conducting automated auctions and transactions. Simulation results show important reductions in utility for sellers under degrading QoS conditions, therefore suggesting the marginal gains of autonomic network support. The results also suggest that observations of the network should be considered in the design of effective trading strategies for such systems.
\end{abstract}

\section{Keywords}

Autonomic networks, distributed systems, automated auctions, electronic commerce, computer simulation.

\section{INTRODUCTION}

Electronic commerce (e-commerce) has evolved quickly from pure electronic transfers within private networks to quite comprehensive systems that can support almost complete business activities on the Internet. Despite the temporal decline of the dot com's a few years ago, electronic commerce continues to be a major business driver, so can be expected that new technological developments will increase e-commerce pervasiveness and position in the global economy.

Autonomic networking is a new technical development that promise improved quality to users and reduced setup

Permission to make digital or hard copies of all or part of this work for personal or classroom use is granted without fee provided that copies are not made or distributed for profit or commercial advantage and that copies bear this notice and the full citation on the first page. To copy otherwise, to republish, to post on servers or to redistribute to lists, requires prior specific permission and/or a fee.

Bionetics '07, December 10-13, 2007, Budapest, Hungary

Copyright 2007 ICST 978-963-9799-11-0. and operation costs to service operators [1]. The implementation of self-ware properties by proper techniques can dynamically configure the network to offer a better service, which would improve quality of service. A related technology are self-aware networks (e.g. cognitive packet networks [9]) that use observations of the network and adaptive routing to dynamically route packets, constantly improving quality-of-service metrics for end-to-end flows.

Given the heterogeneity of the Internet and the development of new technologies that aim at making a more efficient use of network resources for the benefit of users, it is reasonable to inquire about the consequences of such variety of factors in the expected outcome of an e-commerce application. A popular e-commerce application that should be sensitive to network conditions is an auction. The paper considers such case, but unlike current Internet-based systems, such as e-bay or amazon.com auctions, operating under a distributed structure. In a distributed auction, each seller can assume the role of an auctioneer, so that it can directly receive bids from interested buyers (bids are received by a third party in central-based systems). Moreover, the paper considers that auctions will be mainly driven by automated trading agents. There is a wide applicability of the scenario not only to the selling of traditional goods and services, but also as an effective network resource allocation and pricing mechanism.

The distributed structure of the application brings a number of advantages over current centralised structures:

- It easily allows the system to scale to a very large number of participants as bids are not addressed to a single node (or set of nodes) in the network. Therefore, auction load is expected to be reasonably distributed across the network.

- It simplifies the deployment of the system by reducing the equipment and network requirements to system administrators.

- It can be highly available at low cost.

- It prevents participants to expose their trading strategy to a third party, which could deter their profit if misused.

- It makes the system easier to defend against security attacks as there is no single target can be compromised.

On the other hand, a total distributed structure of the system may bring other security issues that could make it 
harder to control, so that a hybrid structure would be more appropriate in this context. That is, the system would allow centralism for general operations, such as marketing and user authentication, authorization and access, but keeping auctions distributed. Nevertheless, it is not illogical to expect a reduction in information centralism as new technologies develop.

Recent papers have addressed the applicability and design of distributed auctions in the context of computer networks and communication networks in general. There is a wide application range for auctions as a resource allocation mechanism. Esteva [3] suggested a distributed architecture for electronic auctions and specified protocols for a firstprice, second-price, Dutch and English auctions by means of $\pi$-calculus. Ezhilchelvan and Morgan [4] developed an alternative distributed system for Internet-based auctions. Hausheer and Stiller have introduced "PeerMart" [10], a system that uses a double auction on a structured P2P overlay network to distribute brokering load for pricing various goods and services (e.g. bandwidth pricing [11]). Franklin and Reiter [6] developed a distributed system for sealed-bid auctions.

Auctions in mobile networks have been studied by Frey et al. [7], Wu et. al in the form of a continuous double auction as part of a routing algorithm [15] and Fourati and Al Agha $[5]$.

The application of auctions for bandwidth allocation have been considered by Dramitinos et al. [2] for 3G networks with generalised automated Vickrey auctions. Also, Li and Mahanti [14] suggested the use of auctions to coordinate streaming sessions.

\section{SYSTEM OF DISTRIBUTED AUCTIONS}

Consider an auction system supported by a computer network [12], where an auction application running in selected nodes enables setting up one or more auction elements (bidders or auctioneers). These elements can interact each other through the network. Interested buyers take the place of bidders that will compete with others for goods or services offered by sellers via auctions. To clarify the use of terms in this paper, we use "seller" to indicate the person or program that decides the selling of some good or service, while "auctioneer" refers to the computing entity able to handle bids and decide acceptance. In general, we consider that auctions will be automated so that their duration could be very short. In a similar way, we differentiate "buyers" from "bidders".

In addition to these elements, there are special nodes in the network that host "auction centres". Such centres allow sellers to advertise their goods and buyers to find potential sellers. Auction centres do not play an active role in the progress of an auction. They simply serve as central advertisement points in the network for the system. In addition, they would allow user registration, supervision and trade settlement (e.g transfer of goods, payments).

\subsection{Auctioning Protocol}

The auctioning protocol used in this study is described by the finite states machines (FSM) depicted in Figures 1,2 and 3 . The protocol extracts the main aspects of a full auctioning system. Supervision and security functionalities have not been included as they are not directly related to the study. The FSMs correspond to the auction centre, auctioneer and bidder respectively. The transitions indicate the packet exchange involved in the corresponding operation with packet names in capital letters.

Sellers advertise items with ADV packets, which contain at least the item description and auction model. This paper considers only English auctions, so that the initial price is also included in the ADV packet. After receiving an ADV, the auction centre will append the item to its list of available items with a reference to the seller address. Bidders interested in the item may send a QRY packet to a centre, which will reply with a list of items matching the request in a RPY packet. Once received, the bidder will select the most appropriate offer from the list if any (e.g. the lowest priced item) and place a bid (BID packet) with the corresponding seller. In addition to serving as the initial contact point, the $\mathrm{AC}$ also keeps information about auctions in progress for new bidders that may wish to join later later. As mentioned before, the interaction of sellers and bidders is fully distributed and occur without direct intervention of the AC.

Auctioneers enter the "Auction" state (Figure 2) after receiving a first bid. After that, they may decide to conclude the auction if no further bids are received within a designated period of time (English auction). If another bid is received before the designed time, the item's price is updated with the newest offer and both previous bidders and AC are updated with the newest price (with HBID and ADVUPD packets respectively). English auctions require each bidder to "hear" all other bids. A bidder that it is not the highest bidder may send a new bid some time after receiving a HBID packet and according to its trading strategy. The trading strategy may dictate, for example, that new bids can be generated only if the current value of the auction is below a pre-calculated threshold. Bidders may withdraw from the auction as long as they are not the highest bidder (by stopping bidding and sending a WDRW packet to the auctioneer).

The process continues iteratively until the auctioneer decides to terminate the auction. Termination occurs according to the auctioneer's trading strategy. Under English auction rules, the auctioneers wait for further bids within a specified period. If no bids are received by then, they conclude the auction and send an WBID packet to all bidders (including the highest bidder) and ADVUPD to the auction centre.

To ensure consistency, the protocol includes a number of timeout mechanisms that can return an auction element to the STOP state is no messages are received to produce normal transitions to other states. Such situations may occur because unexpected situations, such as computer or network malfunction. They may occur also under no demand, for example, an auctioneer not receiving a first bid.

The protocol assumes the use of a connectionless protocol (i.e. UDP) because of the near real-time requirements of the application. Therefore, network latency may cause reception of unexpected messages. Auctioneers may receive delayed bids with a lower or equal price to the current value or after termination of the auction. Similarly, bidders may receive out-of- sequence price updates (HBID). Although not shown explicitly in the FSM, to keep simple the presentation of the protocol, the reception of unexpected packets, which are packets not shown in the transitions, cause no effect and no particular action is taken in the corresponding auction element. 


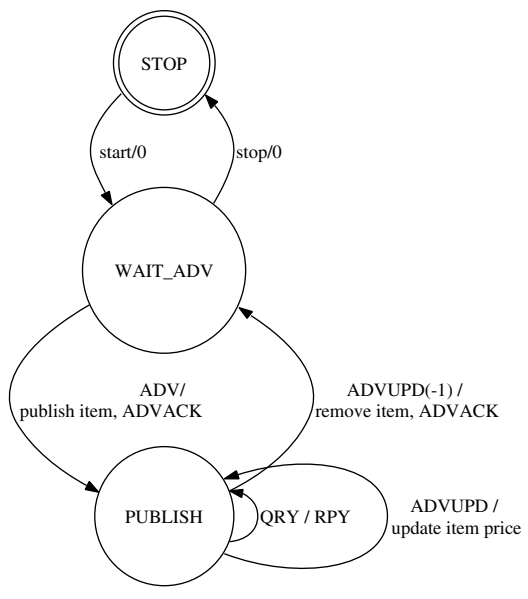

Figure 1: Finite state machine of auction centre.

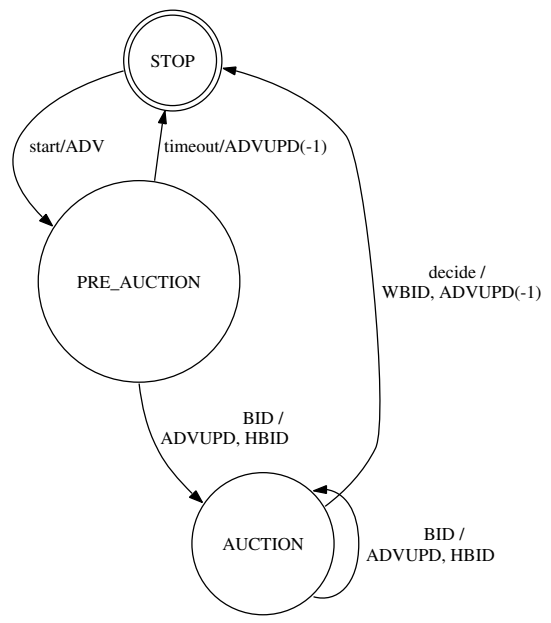

Figure 2: Finite state machine of auctioneer.

\section{SIMULATION}

The simulation setup consisted of a peer-to-peer network connecting the auction elements (bidders, sellers and auction centre). A simulation module of the auctioning protocol described in section 2.1 was developed and integrated into the discrete-event simulator INES [13] to evaluate significant parameters of a distributed auction (Figure 4).

The setup considered the case of a single $\mathrm{AC}$ with bidders and sellers with prior knowledge of the AC address. After starting, the seller announces the (unique) good to sell to the $\mathrm{AC}$ with an initial price of zero (asking price of one). Bidders start at a random time between $100 \mathrm{~ms}$ and $1 \mathrm{~s}$ after the starting time of the seller. Immediately after starting, bidders send a QRY packet to the AC inquiring for the good. Bidders start with a random estimation of the value of the good, which lies uniformly between 80 and 100. If the price surpasses their estimation during the auction, the bidder withdraws the auction. A bid is placed at the expiration of a timer with an exponentially generated interval (parameter b) that is started upon arrival of a reply from the $\mathrm{AC}$ for a good or an HBID message (out-bid message) from the seller.

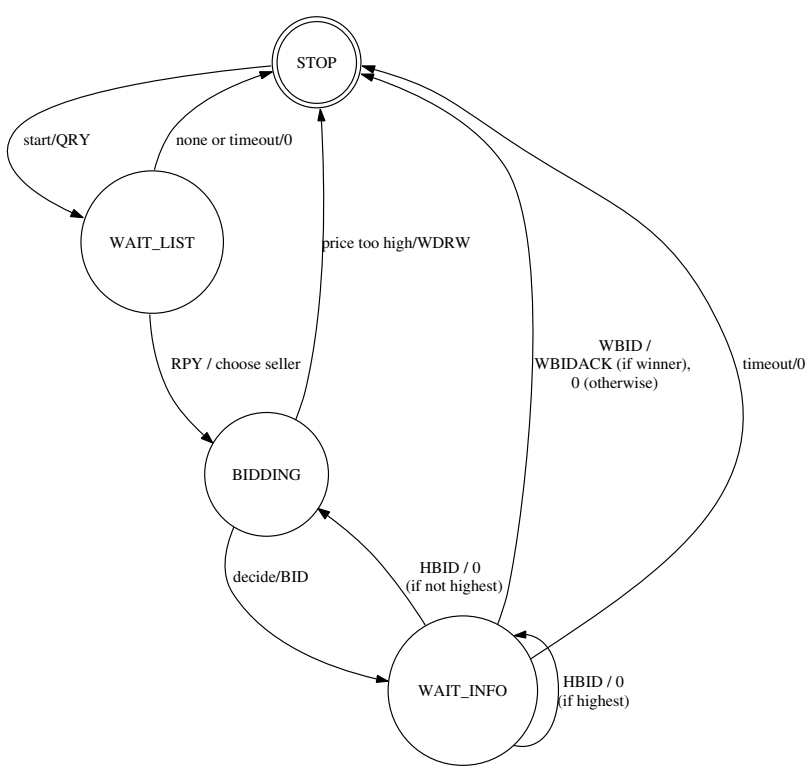

Figure 3: Finite state machine of bidder.

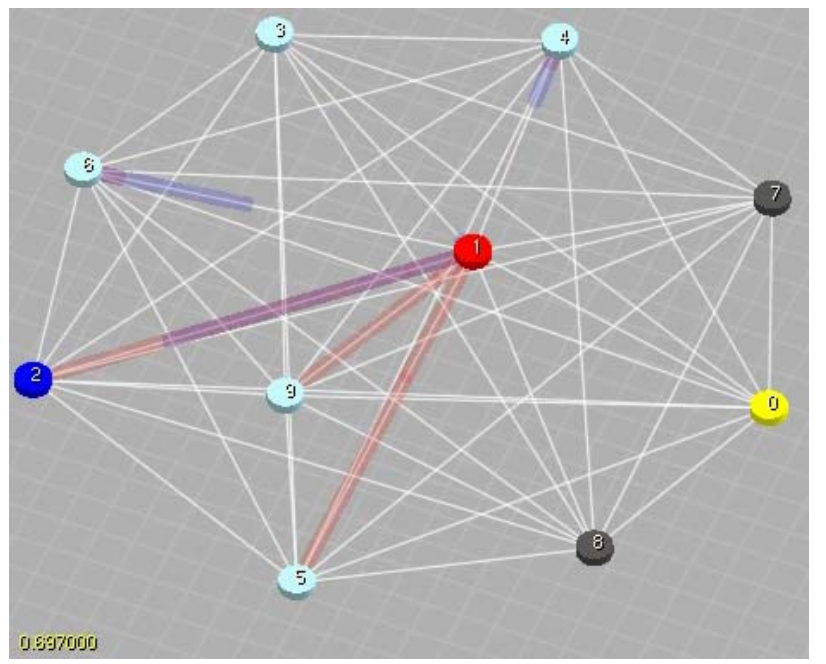

Figure 4: Simulation of a distributed auction system.

A bid always increments in one the current (known) value of the good. The parameter $b$ was kept common to all bidders in the simulations.

Similarly, the seller may decide to accept a bid and terminate the auction upon expiration of a timer started after the reception of a bid (again, with an exponentially distributed time with parameter $d$ ).

Both $b$ and $d$ can model part of the strategy and responsiveness of bidders and sellers. For instance, they could account, among other things, for the valuation time to verify the price of the item in the market. This paper focuses mainly on the sellers' side, so that it is of interest investigating its expected income per unit of time $(\Phi)$. $\Phi$ is the accepted bid divided by the auction duration. The experiment follows closely Gelenbe's evaluation of an analytic model [8] 
but in a simulated network setting with variable QoS conditions.

\subsection{Bandwidth, Loss and $\Phi$}

First, we evaluate the effect of the average end-to-end bandwidth of the network in the seller's expected income per unit of time. For this, we vary the expected bandwidth of the overlay between $64 \mathrm{Kbps}$ to $8 \mathrm{Mbps}-\mathrm{a}$ typical range in today's Internet, for a range of selling decision rates. The bidder decision rate was fixed to $64 \mathrm{bid} / \mathrm{sec}$. Figure 5 shows the simulation results, relating $\Phi$ vs. the seller's decision rate for the selected range of network bandwidth. As shown in the figure, bandwidth can affect greatly the expected income of the seller, which could make up a difference of up to five times within the selected range.

It is interesting to note that although sellers could modify their strategy to achieve maximum profit, they would need know the current network conditions. In the results, the maximum value clearly depends on the bandwidth available.

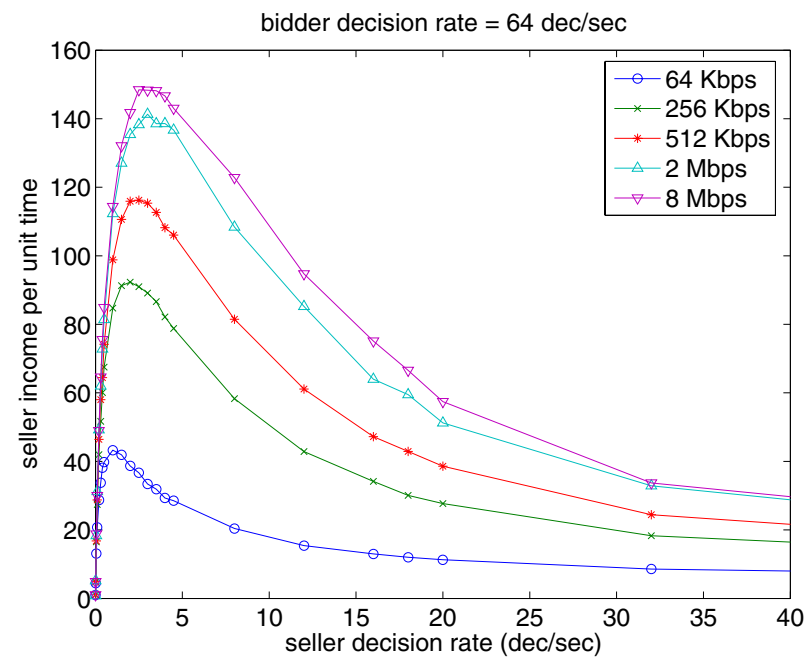

Figure 5: Latency on the income per unit time vs. seller's decision rate.

Figure 6 shows the simulation results of a similar experiment to determine $\Phi$ vs. seller's decision rate, but this time keeping constant network bandwidth (64 Kbps) and varying the end-to-end packet error ratio (PER) of the end-to-end communications.

\subsection{Bid Efficiency}

In addition to decreasing the expected seller's income, longer network delays produce larger numbers of "short" bids, that is, bids with an offer not sufficiently high. These short bids are generated because network delays cause an inevitable time interval between the arrival of a valid bid at the seller and the arrival of the HBID message at bidders which inform them of the current price. Therefore, during that interval, bids will be generated with an incorrect notion of the current price creating the consequent short bids (given that we assume unit increments). We differentiate short bids from "late bids". The latter referring to bids received after termination of the auction. Both short and late bids waste bandwidth and other resources and should be avoided by

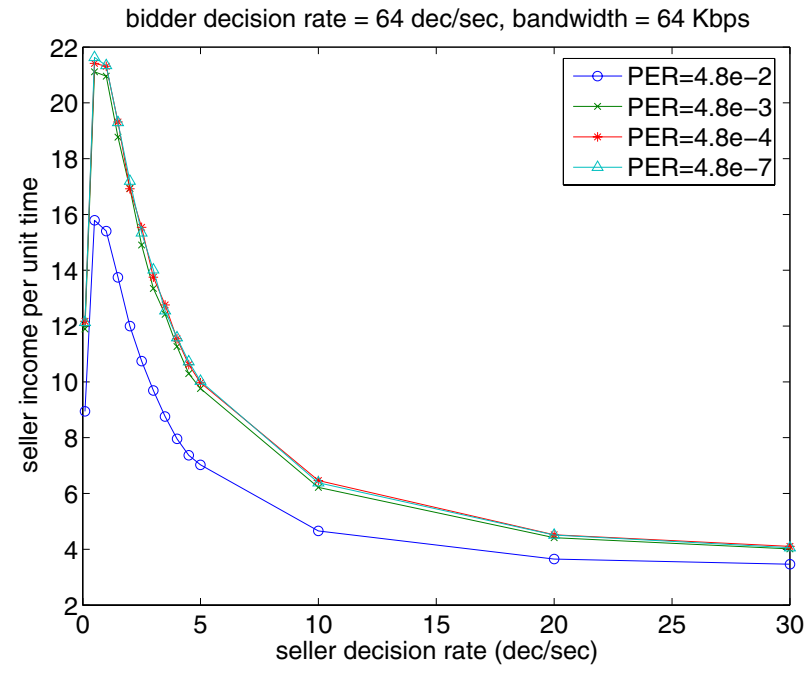

Figure 6: Packet loss on the income per unit time vs. seller's decision rate.

the network and protocol design as possible. We define "bid efficiency" $(\Lambda)$ to the ratio of valid bids (bids arriving at the seller with a correct price) over the total number bids received. Figure 7 depict the measured bid efficiency vs. the seller's decision rate for a bidders' decision rate of 64 bid/sec as before. As expected, bid efficiency increases with network bandwidth.

A small depression can be seen in the figure, around values matching the seller's decision rate, which can be explained by noting that longer decision times cause more bidders to withdraw the auction; therefore, increasing the efficiency of single bids. On the other hand short decisions prevent further bids to be generated (both valid and short ones).

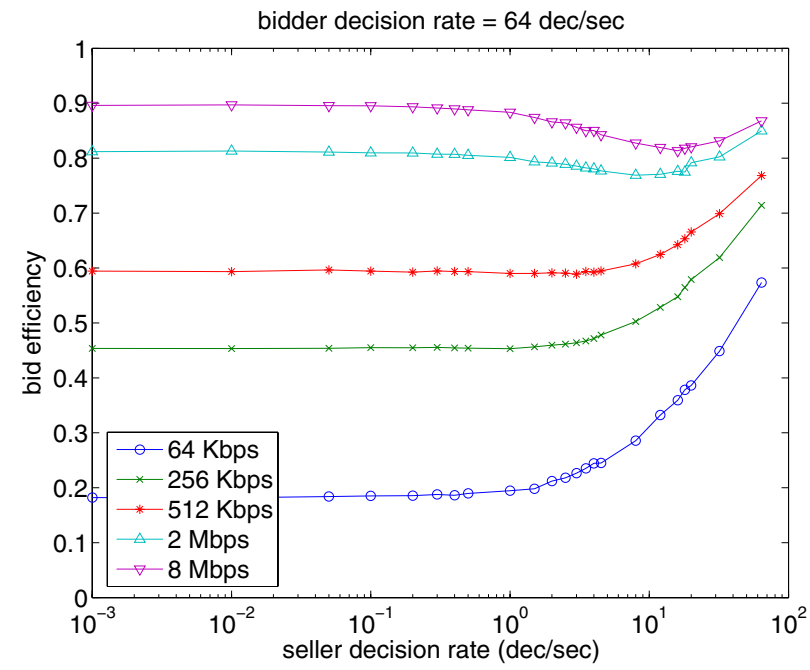

Figure 7: Latency on bid efficiency vs. seller's decision rate for a bidding rate of 64 .

If we repeat the simulation but introducing losses into the system, we could observe an opposite effect. Losses tend to 
increase the bid efficiency because the number of invalid bid are generally higher than valid bids, so they are more prone to be dropped. This effect can be observed in Figure 8. Increasing losses do not produce the significant impact that can be observed with increasing latency, because would-be invalid bids replace valid ones that were dropped.

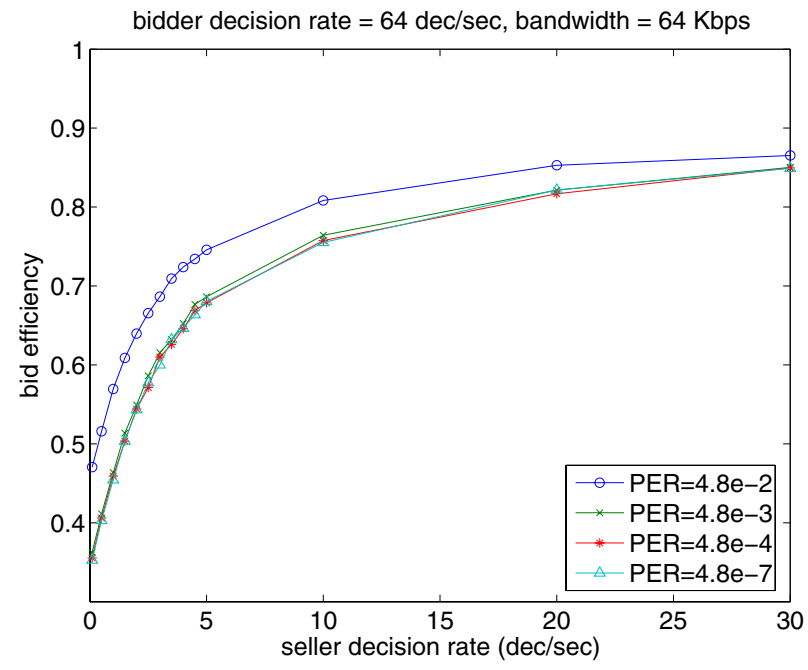

Figure 8: Packet loss on bid efficiency vs. selling decision rate.

\subsection{Protocol Efficiency}

To study the efficiency of the protocol in Section 2.1, we have looked at the total traffic generated by an auction and compared it to the income generated to the seller. The results for the selected bandwidth range are reported in Figure 9. Figure 10 depicts the case with varying packet loss rates. As expected, higher efficiency, from the income point of view, can be observed when faster and more reliable communications are available.

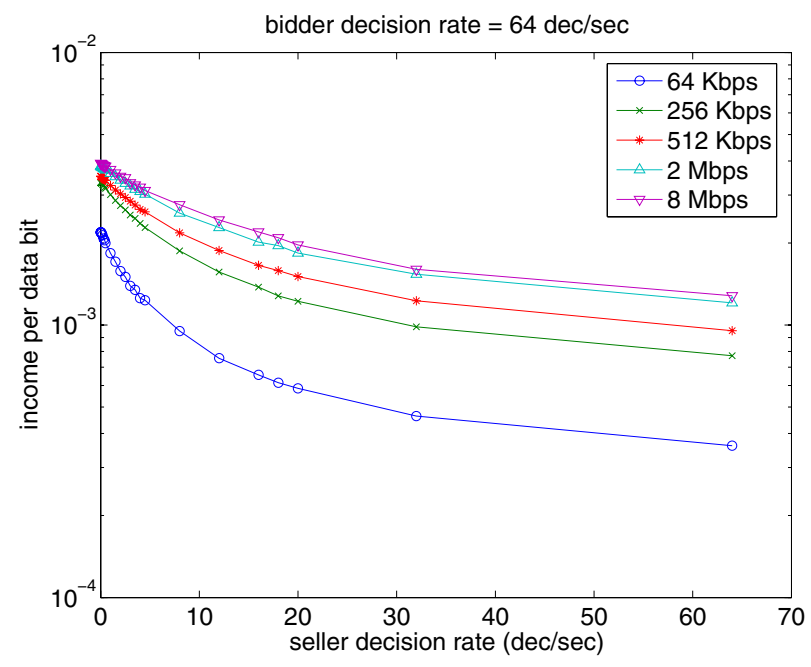

Figure 9: Latency on protocol efficiency vs. selling decision rate.

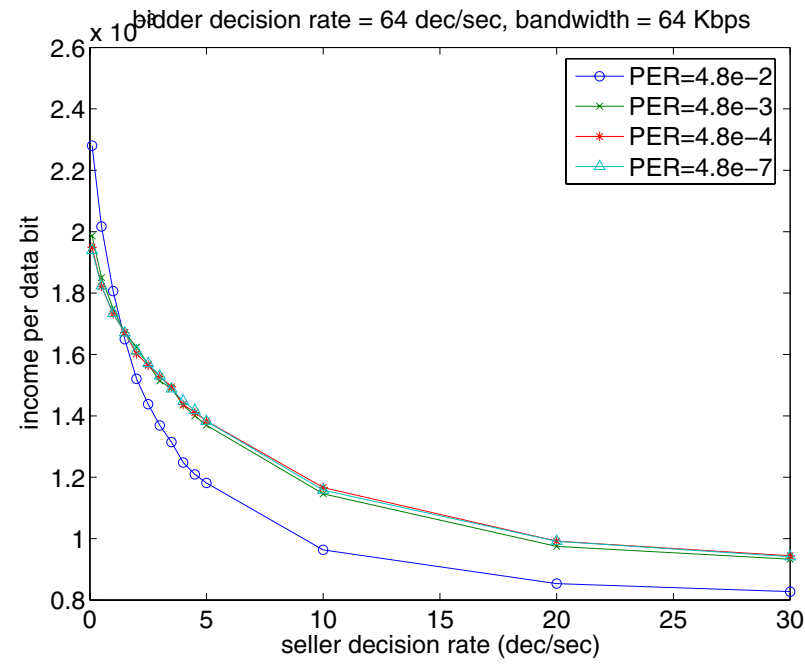

Figure 10: Packet loss on protocol efficiency vs. selling decision rate

\section{CONCLUSIONS}

Autonomic networks are a promising technology that will introduce facilities and improvements to network operators and users alike. With autonomic networks, the system configuration may change to compensate for service degradation with little or no human intervention. Network QoS is the direct result of the system and its configuration, as well as existing network conditions, so it to be expected that autonomic networks will produce significant improvements in QoS compared to more traditional networks. The paper has studied the impact of network QoS on the operation and outcome of a system of distributed auctions to quantify the probable benefits to be obtained with the introduction of autonomic technology in the supporting network. For this purpose, an auction protocol was designed and developed in a computer simulator, along with trading agents for automated operation. The results have shown that increased network latency can significantly reduce the expected income per unit time of sellers and that efficiency, of both the protocol and bids, can be greatly affected. Higher delays induce bidders to generate a larger number of incorrect bids that waste network resources. On the other hand, it was shown that packet losses can also negatively impact an electronic auction, although, to much lesser extend that network latency. The results have determined than the maximum income per unit of time is a function of network QoS, therefore, trading strategies should aim at considering observations of the network in their decisions. Finally, the work suggests that autonomic networks may bring meaningful utility benefits to sellers of automated auctions.

\section{ACKNOWLEDGMENTS}

The work presented in this paper was partially supported by the project CASCADAS (IST-027807) funded by the FET Program of the European Commission. The paper represents the work and contribution of an individual party involved in the project. The author would also like to thank 
the project partners for their excellent comments and discussions.

\section{REFERENCES}

[1] S. Dobson, S. Denazis, A. Fernández, D. Gaïti, E. Gelenbe, F. Massacci, P. Nixon, F. Saffre, N. Schmidt, and F. Zambonelli. A survey of autonomic communications. ACM Trans. Auton. Adapt. Syst., 1(2):223-259, 2006.

[2] M. Dramitinos, G. Stamoulis, and C. Courcoubetis. Auction-based resource allocation in umts high speed downlink packet access (hsdpa). In Next Generation Internet Networks, pages 434-441, April 2005.

[3] M. Esteva and J. A. Padget. Auctions without auctioneers: Distributed auction protocols. In Agent Mediated Electronic Commerce (IJCAI Workshop), pages 220-238, 1999.

[4] P. Ezhilchelvan and G. Morgan. A dependable distributed auction system: Architecture and an implementation framework. In Proceedings of the Fifth International Symposium on Autonomous Decentralized Systems, 2001.

[5] A. Fourati and K. A. Agha. Deploying auctions over ad hoc networks. In ICEBE '06: Proceedings of the IEEE International Conference on e-Business Engineering, pages 9-16, 2006.

[6] M. K. Franklin and M. K. Reiter. The design and implementation of a secure auction service. IEEE Trans. Softw. Eng., 22(5):302-312, 1996.

[7] H. Frey, J. Lehnert, and P. Sturm. Ubibay: An auction system for mobile multihop ad-hoc networks. In Workshop on Ad hoc Communications and Collaboration in Ubiquitous Computing Environments (AdHocCCUCE'02), 2002.
[8] E. Gelenbe. Analysis of automated auctions, keynote. Proceedings. Lecture Notes in Computer Science, 4263, 2006.

[9] E. Gelenbe, R. Lent, and Z. Xu. Measurement and performance of cognitive packet networks. $J$. Computer Networks, 37:691-701, 2001.

[10] D. Hausheer and B. Stiller. Decentralized auction-based pricing with peermart. In 9th IFIP/IEEE International Symposium on Integrated Network Management (IM 2005), May 2005.

[11] D. Hausheer and B. Stiller. Peermart: Decentralized auctions for bandwidth trading on demand. ERCIM News, 68:42-43, Jan 2007.

[12] M. Kumar and S. I. Feldman. Internet auctions. In WOEC'98: Proceedings of the 3rd conference on USENIX Workshop on Electronic Commerce, pages $5-5,1998$

[13] R. Lent. Integrated Network-Environment Simulator (INES). online: http://san.ee.ic.ac.uk/ines.

[14] Z. Li and A. Mahanti. A progressive flow auction approach for low-cost on-demand $\mathrm{p} 2 \mathrm{p}$ media streaming. In QShine '06: Proceedings of the 3rd international conference on Quality of service in heterogeneous wired/wireless networks, page 42, 2006.

[15] Z. Wu, Z. Chen, L. Xu, and F. Guo. Routing protocols based on double auction for ad hoc networks. In International Conference on Networking, Architecture, and Storage (NAS 2007), pages 55-61, 2007. 